\title{
Einstein Ring Constraints on the Shapes of Dark Matter Haloes
}

\author{
Randall B. Wayth ${ }^{1}$, and Rachel L. Webster ${ }^{1}$ \\ ${ }^{1}$ School of Physics, University of Melbourne, VIC 3010, Australia. email: \\ rwayth,rwebster@physics.unimelb.edu.au
}

\begin{abstract}
The shape and size of a galaxy-mass dark matter halo can only be measured using a luminous tracer, such as stars, gas or photons. In the last case, gravitational lensing of extended background sources provides many paths for photons to sample the gravitational potential of the lensing galaxy. Multiple independent light paths provide tighter constraints on the mass profile of the lens galaxy than is possible from single path sampled by the lensed images of unresolved sources.

Using software specifically developed to model resolved images, we analyse the optical and radio Einstein ring systems 0047-2808 and MG1549+3047. For 0047-2808, elliptical pseudoisothermal models (e.g. SIE, PIEP) are able to reproduce the lensed image well, with a surface mass power-law $\Sigma \propto r^{-\gamma}$, best fitted by $\gamma=1.07 \pm 0.03$, which is slightly steeper than isothermal. Using a stars-plus-halo model, we find the projected halo is rounder than the visible galaxy (axis ratio $0.82 \pm 0.01$ ) and aligned closely with the stars (offset $8^{\circ} \pm 1$ ). Preliminary results for MG1549+3047 also rule out the constant M/L model. A stars + PIEP model suggests a very round (axis ratio $\geqslant 0.98$ ) halo.
\end{abstract}

\section{Introduction}

Galaxies comprising baryons and dark matter, are hard to model and the effects that baryons have on the dark matter are not well understood. Numerical models (e.g. Evrard et al. 1994; Tissera \& Dominguez-Tenreiro 1998; Kazantzidis et al. 2004) show that galaxy formation models which include gas cooling produce significantly different dark haloes compared to adiabatic collapse models. Hence, observational techniques for measuring the properties, and therefore testing the models of dark haloes are essential.

In this paper we show that strong gravitational lensing of resolved sources can provide tight constraints on the shape and orientation of dark haloes in the important region where the mass distribution makes the transition from baryon or star-dominated to dark matter dominated. Software (Wayth \& Webster 2004) which has been specifically developed by Wayth for resolved images is used in this analysis.

\section{2. $0047-2808$}

0047-2808 (Warren et al. 1996, 1999) is a $z=3.6$ Ly $\alpha$ emitter lensed into several arcs and bright spots by an elliptical galaxy at $z=0.49$. The lens galaxy is appears to be isolated, with the nearest projected galaxy $\sim 1$ arcmin away. Hence, there is no evidence that the light path is significantly perturbed by external masses. The lens galaxy was fitted with a Sersic light profile (defined by $\Sigma=\Sigma_{1 / 2} \exp \left\{B(n)\left(r / r_{1 / 2}\right)^{1 / n}-1\right\}$ ) with $R_{1 / 2}=1.09^{\prime \prime}$, axis ratio $q=0.69, \mathrm{n}=3.11$ and PA $62.4^{\circ}$, as described in Wayth et al. (2004). The lens galaxy was then subtracted to leave the ring. Figure 1 shows the HST F555W image of 0047-2808 with the lens galaxy subtracted. 

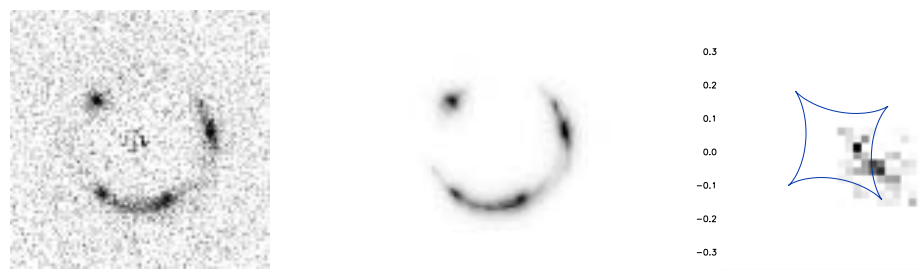

Figure 1. PIEP models for 0047-2808. Left: the $93 \times 93$ pixel frame used in models. The image is $4.65^{\prime \prime}$ on the side. Centre: the image produced by the PIEP mass model. Right: the reconstructed source and caustic. Note the size of the source is much smaller than the observed images, the caustic is approximately $0.3^{\prime \prime}$ across.

Firstly, we model the galaxy's total mass profile using a softened power-law elliptical mass distribution (SPEMD) with surface density $\Sigma \propto r^{-\gamma}$. The parameters of the $\operatorname{SPEMD}$ are mass scale $(b)$, axis ratio $(q)$, orientation angle $(\theta)$ and mass power-law $(\gamma)$. The centre of the lens is fixed to the centre of the light profile. The power-law measures the slope of the mass profile around the region of the images, which are located at $r \sim 7 h_{70}^{-1} \mathrm{kpc}$ for $0047-2808$.

Results of the modelling show a well constrained mass profile, as shown in Figure 2. The mass slope is not strongly degenerate with ellipticity - a common problem associated with fitting multiply-imaged QSOs. The best fitting slope is $\gamma=1.07 \pm 0.03$, slightly steeper than isothermal. The position angle is $\theta=66^{\circ} \pm 1$ (misaligned by $4^{\circ}$ to the galaxy PA), and axis ratio $q=[(1-\epsilon) /(1+\epsilon)]^{3 / 2}=0.77 \pm 0.01$ where $\epsilon$. Since the axis ratio of the total mass distribution $(q=0.77)$ is greater than the stellar light profile $(q=0.69)$, the halo must be rounder than the stars.

With the scale, ellipticity, position angle and slope of the total mass profile well constrained from the image data alone, we focus now on separating the properties of the halo from the visible matter. We use a stars-plus-halo model for the overall mass distribution in the lens galaxy. For the stars, we use the measured light profile with stellar $\mathrm{M} / \mathrm{L}, \Upsilon$, as a free parameter. For the halo we use a pseudo-isothermal elliptic potential (PIEP, defined by the lensing potential $\left.\psi=b\left[x^{2}(1-\epsilon)+y^{2}(1+\epsilon)+r_{c}^{2}\right]^{1 / 2}\right)$ with constant surface density core of scale length $r_{c}$. The PIEP is not a realistic model for the halo far from the galaxy because the mass is not finite, but it is perfectly adequate for describing the properties of the halo in the region of the images (although other models are possible). The free parameters of the model are $b, \epsilon, \theta_{\text {halo }}, r_{c}$ (for the halo), and $\Upsilon$ (for the stars).

We find some halo properties are well constrained, but a significant degeneracy exists between $b, \Upsilon$ and $r_{c}$. The halo axis ratio varies between $0.8<q<0.9$ and PA varies between $66^{\circ}<\theta_{\text {halo }}<70^{\circ}$, depending on $r_{c}$. By using additional constraints on $\Upsilon$ such as velocity dispersion (Koopmans \& Treu 2003) or colours from population models, all the properties of the halo in the region of the images can be well constrained.

As an example, we use $\Upsilon=3.1$ which corresponds to $\sim 50 \%$ of the mass inside the images being stellar. We find the following properties of the dark halo of 0047-2808: axis ratio $=[(1-\epsilon) /(1+\epsilon)]^{3 / 2}=0.82 \pm 0.01$, rounder than the stars, and $\theta_{\text {halo }}=70^{\circ} \pm 1$, misaligned by $8^{\circ}$ to the stars. Figure 2 shows confidence contours in $b, \theta_{\text {halo }}$ and $q$ for fixed $\Upsilon=3.1$. Figure 3 shows the image with lens galaxy and the position angles of the various components. It is clear that the projected halo is closely aligned with the stellar surface brightness and is contributing to the shear of the overall mass distribution. 

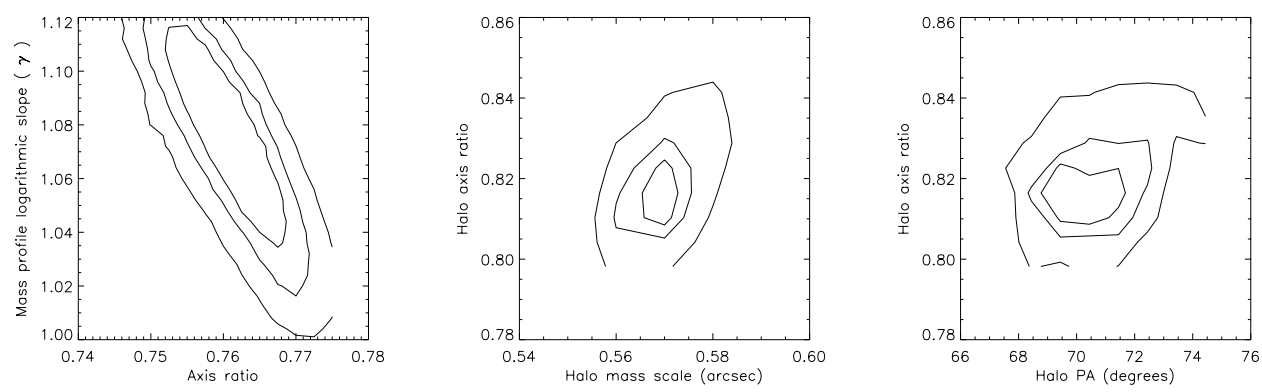

Figure 2. Confidence intervals for models of 0047-2808. Contours are 68\%, 90\% and 99\%. Left: Marginalised confidence intervals for the SPEMD ellipticity vs mass profile. Centre: Marginalised confidence intervals for $b, q$ in the stars-plus-halo model with fixed $\Upsilon=3.1$. Right: Marginalised confidence intervals for $\theta_{\text {halo }}, q$ in the stars-plus-halo model with fixed $\Upsilon=3.1$.

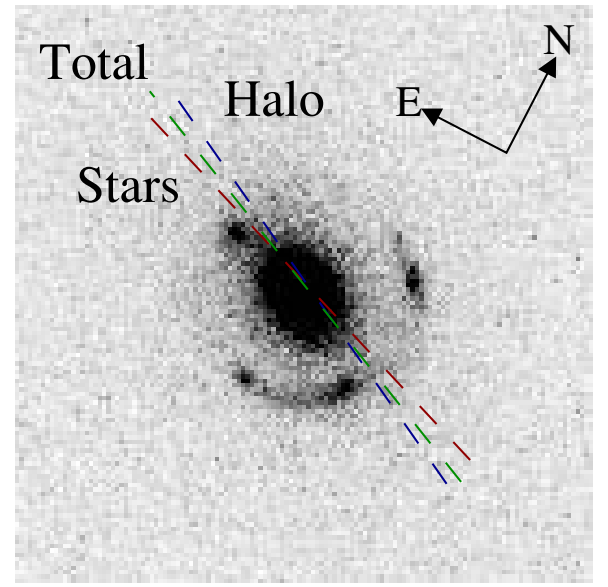

Figure 3. HST F555W image of 0048-2708 with the position angles of the various mass models. Shown is the PA of the stars, PIEP total mass model and PIEP halo model.

\section{MG1549+3047}

MG1549+3047 (Lehar et al. 1993) is a radio lobe produced by a QSO at $z=1.17$ (Treu \& Koopmans 2003) lensed into a ring by a $z=0.111$ SB0 galaxy. The lensing galaxy has a prominent bulge, faint bar and very faint disk.

Prior to this work, this system was modelled by identifying features in the ring which were likely to be images of the same part of the source radio lobe. The best PIEP-style lens model found by Lehar et al. (1993) has parameters $b=1.15^{\prime \prime}, \epsilon=0.073, r_{c}=0.22^{\prime \prime}$ and $\mathrm{PA}=-48^{\circ}$.

Here we present a preliminary analysis of MG1549+3047 using archival VLA $3 \mathrm{~cm}$ data. The best fitting PIEP model $\left(\chi^{2} / \nu=1.15\right)$ has parameters $b=1.10^{\prime \prime}, \epsilon=0.054$, $\theta=-47^{\circ}$ and $r_{c}=0.12^{\prime \prime}$. The data and best-fitting model are shown in Figure 5 and the reconstructed radio lobe and counterpart unlensed lobe are shown in Figure 6 . The lobe's brightest region is just outside the multiple image region of the lens, so the image is sharply truncated at the centre of the lens. Hence, the location of the centre of the lens must be determined accurately. We mention also that a constant $\mathrm{M} / \mathrm{L}$ model is a very poor fit $\left(\chi^{2} / \nu=2.76\right)$, so a halo is required to explain the lensed image. 


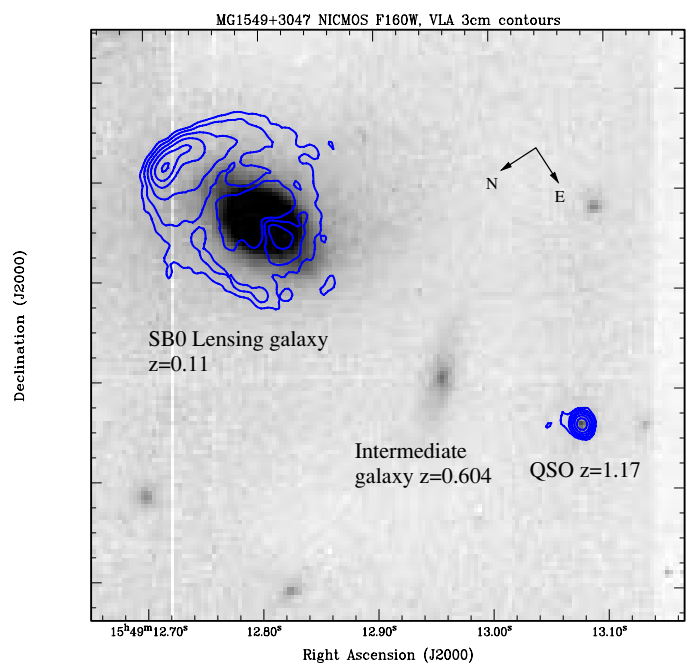

Figure 4. MG1549+3047 QSO, galaxy and lensed lobe. VLA $3 \mathrm{~cm}$ contours are overlaid on the HST NICMOS F160W image. Contours double from $0.2 \mathrm{mJy} /$ beam. The QSO's other (unlensed) lobe is not shown.

Again, we use a stars-plus-halo model consisting of a constant M/L stellar component with a PIEP halo (with constant density core). A realistic constant $\mathrm{M} / \mathrm{L}$ model for the galaxy's stars should contain contributions from the bulge, bar and disk. We found analytic models of the light profile were very sensitive to the size of the region being fitted and had difficulty with the bar. We therefore chose to use the NICMOS F160W image as a direct measure of the stellar surface density. The lensing deflection equation

$$
\overrightarrow{\hat{\alpha}}(\vec{\xi})=\frac{4 G}{c^{2}} \int \frac{\Sigma\left(\overrightarrow{\xi^{\prime}}\right)\left(\vec{\xi}-\overrightarrow{\xi^{\prime}}\right)}{\left|\vec{\xi}-\overrightarrow{\xi^{\prime}}\right|^{2}} d^{2} \xi^{\prime}
$$

can be viewed as a convolution of the surface mass density with the vector $1 / \vec{\xi}$. Convolutions can be easily implemented via Fourier transform, so we chose this approach to calculate the deflection due to the stars in the lens galaxy. We calculated the deflection angle at each NICMOS pixel by multiplying the Fourier transforms of appropriately shifted and padded images of the galaxy and $1 / \vec{\xi}$, then by taking the inverse transform. Our software uses these deflections directly and interpolates between pixels where necessary.

The best fitting result has an almost circular $(\epsilon \leqslant 0.02)$ halo with large $\left(r_{c}=2^{\prime \prime}\right)$ core. The position angle is not well constrained because the halo is so round, but the best fit is for $\theta=10^{\circ}$, which is perpendicular to the PA of the galaxy bulge and also (coincidentally) perpendicular to the PA of an external galaxy, which is close to the line-of-sight. We interpret this as an indication that the halo model is compensating for an excess of shear along the PA of the galaxy bulge. It is possible that this system will require a more complex halo model because the image of the lobe spans $2^{\prime \prime}$ of the galaxy's radius, including the very centre. The mass profile of the halo could reasonably be expected to vary considerably over this range in a way that is not well described by the cored PIEP model. Nevertheless, it is clear that in the case of MG1549+3047, the galaxy bulge is contributing virtually all of the shear required to explain the lensed image and any halo model is likely to have very small ellipticity. 

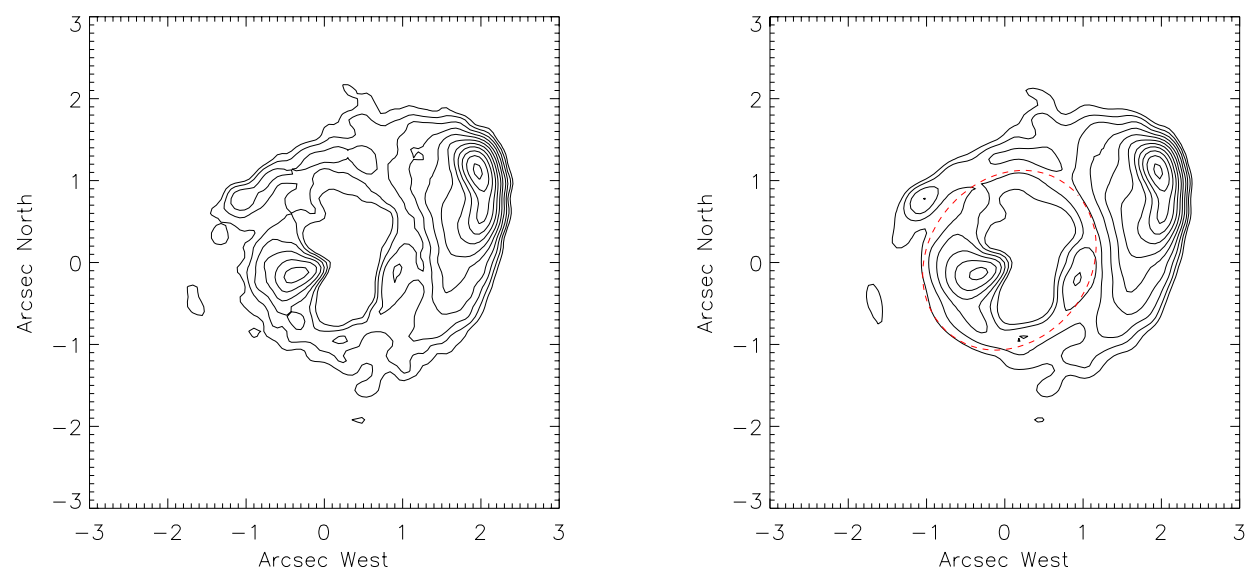

Figure 5. Data (left) and model image with critical line (right) the MG1549+3047 PIEP lens model. Contours increase by $\sqrt{2}$ from $0.2 \mathrm{mJy} /$ beam. The beam size is $0.24^{\prime \prime}$ FWHM.
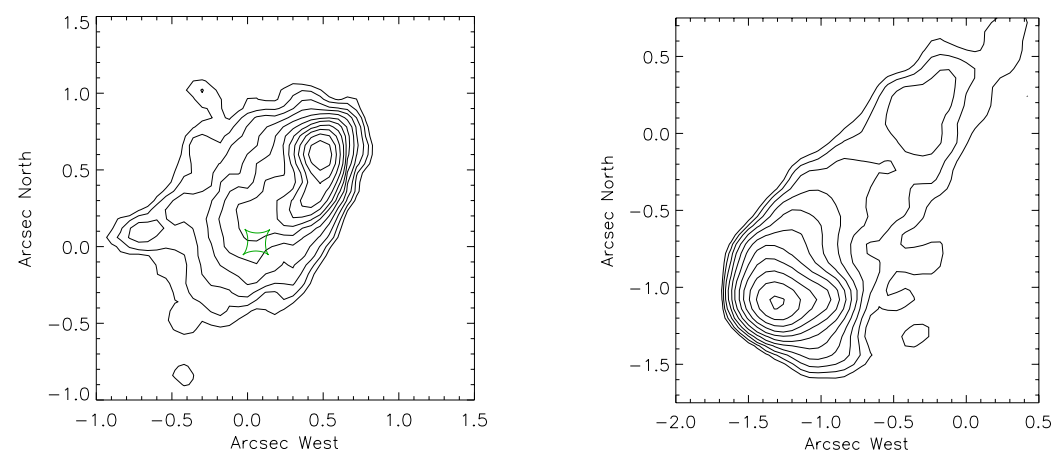

Figure 6. Left: the reconstructed source radio lobe for MG1549+3047 using a PIEP model. Right: the other (unlensed) lobe of the QSO. Contours increase by $\sqrt{2}$ from $0.2 \mathrm{mJy} / \mathrm{beam}$. The unlensed lobe has beam size $0.24^{\prime \prime}$ FWHM.

\section{Conclusions}

We have shown that lensed images of resolved objects permit measurements of the ellipticity and orientation of dark matter haloes in the inner parts of galaxies. Our results for two lensing galaxies show the halo is rounder than the stars in both cases hinting that the baryons can significantly alter the inner regions of haloes compared to expectations from pure $N$-body simulations. A larger sample is needed to quantify this discrepancy. In the case of 0047-2808, the halo is aligned closely with the stars (offset $8^{\circ}$ ). For MG1549+3047, the halo is almost circular.

\section{Acknowledgements}

Thanks to Steve Warren who provided the reduced, lens galaxy subtracted data for 0047-2808. Thanks to Chuck Keeton who gave me the idea to calculate deflection angles by Fourier transform. 


\section{References}

Evrard A. E., Summers F. J., \& Davis M., 1994, ApJ, 422, 11

Kazantzidis S., Kravtsov A. V., Zentner A. R., Allgood B., Nagai D., \& Moore B., 2004, ApJ, 611, L73

Koopmans L. V. E., \& Treu T., 2003, ApJ, 583, 606

Lehar J., Langston G. I., Silber A., Lawrence C. R., \& Burke B. F., 1993, AJ, 105, 847

Tissera P. B., \& Dominguez-Tenreiro R., 1998, MNRAS, 297, 177

Treu T., \& Koopmans L. V. E., 2003, MNRAS, 343, L29

Warren S. J., Hewett P. C., Lewis G. F., Moller P., Iovino A., \& Shaver P. A., 1996, MNRAS, 278,139

Warren S. J., Lewis G. F., Hewett P. C., Møller P., Shaver P., \& Iovino A., 1999, A\&A, 343, L35

Wayth R. B., Warren S. J., Lewis G. F., \& Hewett P. C., 2004, MNRAS, submitted

Wayth R. B., \& Webster R. L., 2004, MNRAS, submitted

\section{Discussion}

J. TAYLOR: I presume the constraints on halo shape are only valid within the Einstein radius. What physical scale does this correspond to?

R. WAYTH: Yes, the strong lensing results are relevant only in the region of the images. For $0047-2808$, that is about $7 \mathrm{kpc}$ from the galaxy centre. For MG1549+3047 that ranges from the very centre of the galaxy to about $2 \mathrm{kpc}$. 